\title{
Prognostic significance of Epstein-Barr virus DNA in NK/T-cell lymphoma: a meta-analysis
}

This article was published in the following Dove Press journal: OncoTargets and Therapy

\author{
Qian Fei* \\ Xiao-Kang Tian* \\ Jing Wu \\ Hong-Ming Zhu \\ Yan Wang \\ Fan-Yu Peng \\ Wen-Jun Zhang \\ Li Yin \\ Xia $\mathrm{He}$
}

Department of Radiation Oncology, Jiangsu Cancer Hospital, Jiangsu Institute of Cancer Research, Nanjing Medical University Affiliated Cancer Hospital, Nanjing, People's Republic of China

*These authors contributed equally to this work
Correspondence: Xia He; Li Yin Department of Radiation Oncology, Jiangsu Cancer Hospital, 42 Baiziting, Nanjing, Jiangsu 210009, People's

Republic of China

Tel +86 I36 $014585 \mid 8$

+86 I39 I390 9043

Email hexiabm@I63.com;

yinli_2012@126.com

\begin{abstract}
Extranodal NK/T-cell lymphoma is closely associated with Epstein-Barr virus (EBV) infection. However, the prognostic value of EBV-DNA in extranodal NK/T-cell lymphoma remains unclear. Thus, we conducted a meta-analysis to estimate its prognostic significance. PubMed, EMBASE, and Web of Science were used to search for studies conducted until June 12, 2017. The pooled hazard ratio (HR) and its $95 \%$ confidence interval (CI) were calculated to evaluate the prognostic value of pretreatment EBV-DNA on the overall survival of extranodal NK/T-cell lymphoma. Seven eligible studies on 356 patients with extranodal NK/T-cell lymphoma were pooled for this meta-analysis. Results suggested that the pretreatment EBV-DNA positivity was significantly correlated with the overall survival of extranodal NK/T-cell lymphoma (pooled HR $=3.78,95 \%$ CI: $1.52-9.40, p=0.004$; heterogeneity test: $I^{2}=52 \%, p=0.05$ ). Subgroup analyses stratified by sample type, survival analysis mode, and HR origin showed that patients with positive pretreatment EBV-DNA had poorer prognosis than those with negative pretreatment EBV-DNA. Moreover, the cut-off value (HR $=1.66$; 95\% CI: $0.73-3.73 ; p=0.22$ ) might account for the heterogeneity. No significant publication bias was observed. Pretreatment EBV-DNA positivity can predict poor prognosis for patients with extranodal NK/T-cell lymphoma. Future large-scale studies based on prognostic significance of EBV-DNA for patients with extranodal NK/T-cell lymphoma are necessary.
\end{abstract}

Keywords: extranodal natural killer/T-cell lymphoma, Epstein-Barr virus, prognosis, meta-analysis

\section{Introduction}

Extranodal natural killer/T-cell lymphoma (NKTCL) is an Epstein-Barr virus (EBV)-associated and highly aggressive non-Hodgkin lymphoma with poor prognosis in advanced patients. ${ }^{1}$ NKTCL is infrequent in Western countries but is the most prevalent subtype of peripheral T-cell lymphoma in China, and accounts for $47 \%-56 \%$ of peripheral T-cell lymphoma and $10 \%-15 \%$ of non-Hodgkin lymphoma. ${ }^{2-4}$ Concurrent chemoradiotherapy followed by non-anthracycline-based chemotherapy significantly improves the survival in NKTCL..$^{5-7}$ However, the survival outcome of NKTCL remains poor because of relapse or treatment-related mortality. Therefore, good indicators for overall survival (OS) in NKTCL should be identified. EBV is a ubiquitous $\gamma$-herpes virus that activates infected lymphocyte proliferation through the expression of growth-promoting latency genes and membrane proteins. ${ }^{8}$ The expression of EBV latent genes combined with other cofactors leads to virus-associated tumors such as NKTCL. ${ }^{8}$ EBV-DNA can be detected by polymerase chain reaction (PCR) methods and has been used as a surrogate marker for evaluating tumor load in several EBV-associated malignancies. ${ }^{8-10}$ Recently, the results of some studies showed that pretreatment EBV-DNA levels are correlated with poorer prognosis in NKTCL. ${ }^{11-15}$ 
However, some showed that pretreatment EBV-DNA levels cannot be used as a prognostic factor for NKTCL. ${ }^{16,17}$ The prognostic value of pretreatment EBV-DNA levels remains controversial. Therefore, we conducted a meta-analysis to more precisely evaluate the association between EBV-DNA and the survival outcome of NKTCL.

\section{Methods}

This meta-analysis was conducted according to the Preferred Reporting Items for Systematic Reviews and MetaAnalyses. ${ }^{18,19}$

\section{Search strategy}

Three electronic databases (ie, PubMed, EMBASE, and Web of Science) were searched to examine the studies (published before June 12, 2017) that explored the prognostic value of EBV-DNA on the survival of NKTCL without geographic or language restrictions. Medical Subject Headings words that were used as keywords included the following: "Epstein-Barr virus," "Extranodal NK-T-Cell Lymphoma," and "prognosis." Detailed search strategies that were used in the three electronic databases are described in Table S1. Meanwhile, we carefully screened the references of each retrieved relevant article to find other studies that satisfied the eligibility criteria. Disagreements were settled through discussions within our team.

\section{Selection and exclusion criteria}

The eligibility criteria of literature were as follows: 1) patients with confirmed NKTCL diagnosis according to the World Health Organization classification for lymphomas; 2) the study evaluated the relationship between EBV-DNA and patient OS; 3 ) the study reported a hazard ratio (HR) and 95\% confidence interval (CI) or sufficient data to calculate the HR and $95 \%$ CI according to methods provided by Parmar et al;20,21 4) literature was published in full text. The exclusion criterion was when studies were published as reviews, comments, or conference abstracts.

\section{Data extraction}

Two investigators independently extracted useful data, using a predesigned table, from eligible studies. The following were included in the extracted information: first author's full name, publication year, nationality and ethnicity of study population, number of patients with positive pretreatment EBVDNA and negative pretreatment EBV-DNA, tumor stage, treatment, specimen, method of detection, cut-off value, HR,
95\% CI, and whether multivariate analysis was performed. Disagreements were resolved through group discussion.

\section{Quality assessment}

Two researchers evaluated the study quality according to the Newcastle-Ottawa Scale (Table S2). ${ }^{22}$ The following were included in the main categories used to assess each study: selection, comparability, and outcome. The NewcastleOttawa Scale scores ranged from 0 to 9 , and scores $\geq 6$ indicated high quality. Disagreements were settled through group discussion.

\section{Statistical analysis}

HR was the most appropriate effect measure to analyze time-to-event data. The prognostic value of EBV-DNA on OS was estimated by HR and the corresponding 95\% CI. The most accurate method for determining HR and 95\% CI was to directly retrieve them from the reported results. However, if the study did not report the HR and 95\% CI, these can be calculated by extracting survival rates from OS curves. ${ }^{23}$ Moreover, we defined HR $>1$ as a poor prognosis for the patients with positive EBV-DNA. We used Revman5.3 Software (Review Manager 5 is the software used for preparing and maintaining Cochrane Reviews) to perform the meta-analysis, and the heterogeneity of studies was assessed using Cochrane's Q test ( $\chi^{2}$ test) and $I^{2}$ metric. ${ }^{24}$ $I^{2}<25 \%$ was defined as low heterogeneity, $I^{2}=25 \%-50 \%$ was defined as moderate heterogeneity, and $I^{2}>50 \%$ was defined as significant heterogeneity. ${ }^{25}$ A fixed-effect model was used in the meta-analysis when no significant heterogeneity ( $I^{2}<50 \%$ or $p \geq 0.1$ ) was detected between studies. Otherwise, the random-effect model was selected.

Publication bias was evaluated by Begg's test. KaplanMeier curves were analyzed using Engauge Digitizer version 4.1. Statistical analysis was performed using Stata software (version 12.0; StataCorp., College Station, TX, USA). All $p$-values were two-sided. $p$-values $<0.05$ were considered as statistically significant.

\section{Results}

\section{Characteristics of studies}

A total of 458 articles were selected through searching the databases, and 216 overlapping studies were excluded. After screening the titles and abstracts, 23 studies that might satisfy the inclusion criteria were assessed by reviewing the full texts. As a result, seven eligible studies on 356 patients with NKTCL were included for the meta-analysis (Figure 1). The main characteristics and data of included studies are shown 


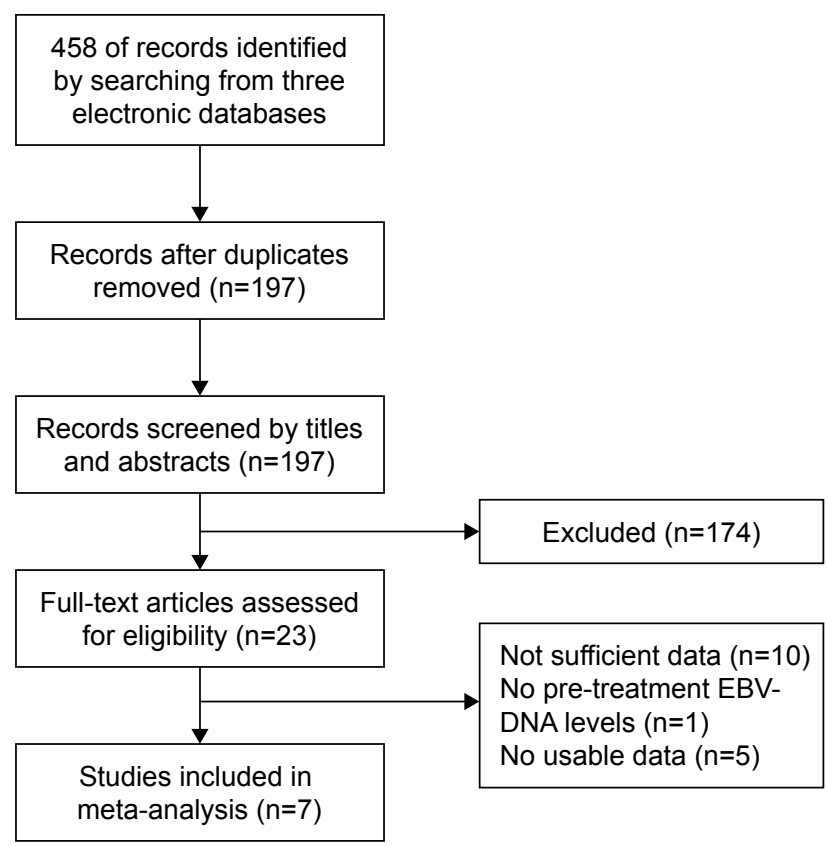

Figure I Flow diagram of study selection for meta-analysis.

Abbreviation: EBV, Epstein-Barr virus.

in Table 1. All of the patients included in our analysis were Asian, and real-time PCR was the method used for EBVDNA detection in all eligible studies. The cut-off values were different within the studies, because the cut-off definition of EBV-DNA has not been standardized. Four studies reported HR with $95 \% \mathrm{CI}$, and the remaining studies calculated HR and 95\% CI from their Kaplan-Meier survival curves. Four studies used whole blood, and the other three studies used plasma due to the lack of consensus on the best blood sample type for EBV-DNA detection.

\section{Prognostic value of pretreatment EBV-DNA in NKTCL}

As shown in Figure 2, the pretreatment EBV-DNA was significantly correlated with the OS of NKTCL patients ( $\mathrm{HR}=3.78,95 \%$ CI: $1.52-9.40, Z=2.85, p=0.004$, randomeffect), and pretreatment EBV-DNA positivity could be used as a predictor for adverse outcomes in NKTCL. The results of Q test and $I^{2}$ test showed a significant heterogeneity across studies ( $p=0.05, I^{2}=52 \%$ ). Therefore, subgroup analyses stratified by sample type, cut-off value, survival analysis mode, and HR origin were further conducted to detect the heterogeneity among eligible studies (Table 2). The results of subgroup analyses, which implied that patients with positive pretreatment EBV-DNA had poorer OS than those with negative pretreatment EBV-DNA, were as follows: whole blood subgroup (HR $=2.15$; 95\% CI: $1.17-3.94 ; p=0.01$ ),

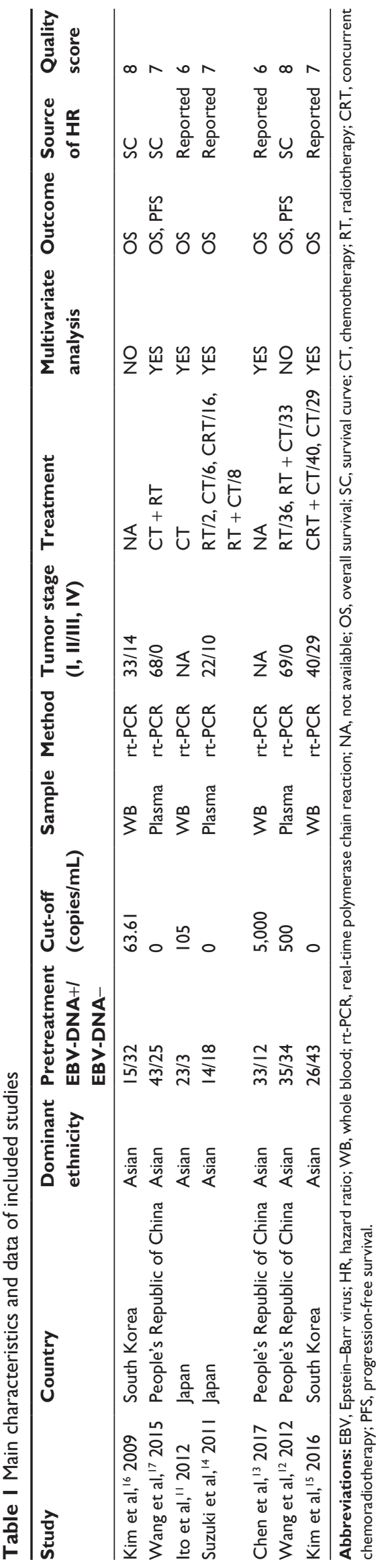




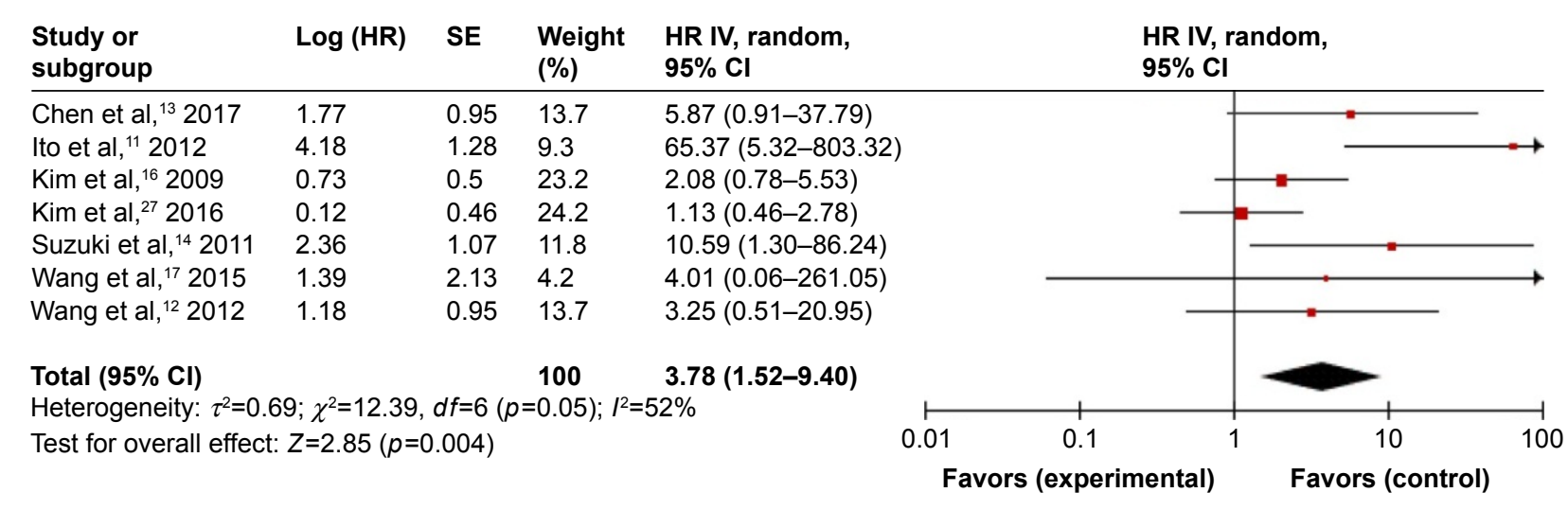

Figure 2 Forest plots for the correlation between pretreatment EBV-DNA and OS.

Abbreviations: EBV, Epstein-Barr virus; OS, overall survival; $\mathrm{SE}$, standard error of the mean; $\mathrm{HR}$, hazard ratio; $\mathrm{Cl}$, confidence interval.

plasma subgroup $(\mathrm{HR}=5.31 ; 95 \% \mathrm{CI}: 1.42-19.88 ; p=0.01)$, cut-off value $>0$ copies $/ \mathrm{mL}$ subgroup ( $\mathrm{HR}=3.60 ; 95 \%$ CI: $1.70,7.62 ; p=0.0008)$, multivariate analysis subgroup (HR $=2.24 ; 95 \%$ CI: $1.05-4.79 ; p=0.04)$, non-multivariate analysis subgroup (HR $=2.86$; 95\% CI: $1.28-6.38 ; p=0.001$ ), HR reported subgroup (HR $=2.65$; 95\% CI: $1.29-5.48$; $p=0.008$ ), and HR calculated from survival curve subgroup (HR $=2.34 ; 95 \%$ CI: $1.00-5.47 ; p=0.05$ ). Moreover, the results of subgroup analyses indicated that the cut-off value (HR $=1.66$; 95\% CI: 0.73-3.73; $p=0.22$ ) might account for the heterogeneity.

\section{Publication bias and sensitivity analysis}

We used Stata12.0 software to conduct Begg's text to estimate the publication bias. As shown in Figure 3, no evidence of publication bias was observed according to Begg's tests ( $p=0.072$ ). Moreover, we performed sensitivity analysis to assess the influence of each individual study on the combined effect size. Results suggested that no individual study significantly influenced our final outcome (Figure 4), which demonstrated that this meta-analysis is relatively stable and statistically reliable.

\section{Discussion}

Meta-analysis is considered as an important tool to objectively evaluate evidence. At present, no systematic review about the prognostic value of EBV-DNA for NKTCL is available. For the first time, this meta-analysis overcame the limitation of sample size, suggesting that the patients with positive pretreatment EBV-DNA had significantly inferior outcome to those with negative pretreatment EBV-DNA.

There are many NKTCL-associated prognostic factors that have been reported so far, such as age, LDH level, Ann Arbor stage, etc. However, in Wang et al's study, ${ }^{14}$ most risk factors of previous prognostic models (Korean Prognostic Index [KPI], International Prognostic Index [IPI]) were found to be unrelated with survival outcomes, indicating the poor survival

Table 2 The prognostic impact of pretreatment EBV-DNA in NKTCL according to subgroup analysis

\begin{tabular}{|c|c|c|c|c|c|c|c|c|}
\hline \multirow{2}{*}{$\begin{array}{l}\text { Subgroup } \\
\text { analysis }\end{array}$} & \multirow{2}{*}{$\begin{array}{l}\text { Number } \\
\text { of studies }\end{array}$} & \multirow[t]{2}{*}{ HR $(95 \% \mathrm{Cl})$} & \multirow[t]{2}{*}{$z$} & \multirow[t]{2}{*}{$p$-value } & \multirow[t]{2}{*}{ Model } & \multicolumn{3}{|c|}{ Test of heterogeneity } \\
\hline & & & & & & $\chi^{2}$ & $p$-value & $I^{2}(\%)$ \\
\hline Overall & 7 & $3.78(1.52-9.40)$ & 2.85 & 0.004 & Random & 12.39 & 0.05 & 52 \\
\hline \multicolumn{9}{|l|}{ Sample } \\
\hline WB & 4 & $2.15(1.17-3.94)$ & 2.48 & 0.01 & Fixed & 10.21 & 0.02 & 71 \\
\hline Plasma & 3 & $5.31(1.42-19.88)$ & 2.48 & 0.01 & Fixed & 0.70 & 0.70 & 0 \\
\hline \multicolumn{9}{|c|}{ Cut-off (copies/mL) } \\
\hline 0 & 3 & $1.66(0.73-3.73)$ & 1.22 & 0.22 & Fixed & 3.88 & 0.14 & 48 \\
\hline$>0$ & 4 & $3.60(1.70-7.62)$ & 3.35 & 0.0008 & Fixed & 6.62 & 0.09 & 55 \\
\hline \multicolumn{9}{|c|}{ Multivariate analysis } \\
\hline Yes & 4 & $2.24(1.05-4.79)$ & 2.09 & 0.04 & Fixed & 10.28 & 0.02 & 71 \\
\hline No & 3 & $2.86(1.28-6.38)$ & 2.57 & 0.001 & Fixed & 1.93 & 0.38 & 0 \\
\hline \multicolumn{9}{|l|}{ Source of HR } \\
\hline SC & 3 & $2.34(1.00-5.47)$ & 1.96 & 0.05 & Fixed & 0.24 & 0.89 & 0 \\
\hline Reported & 4 & $2.65(1.29-5.48)$ & 2.64 & 0.008 & Fixed & 12.10 & 0.007 & 75 \\
\hline
\end{tabular}

Abbreviations: EBV, Epstein-Barr virus; NKTCL, extranodal natural killer/T-cell lymphoma; HR, hazard ratio; Cl, confidence interval; WB, whole blood; SC, survival curve. 


\section{Begg's funnel plot with pseudo $95 \%$ confidence limits}

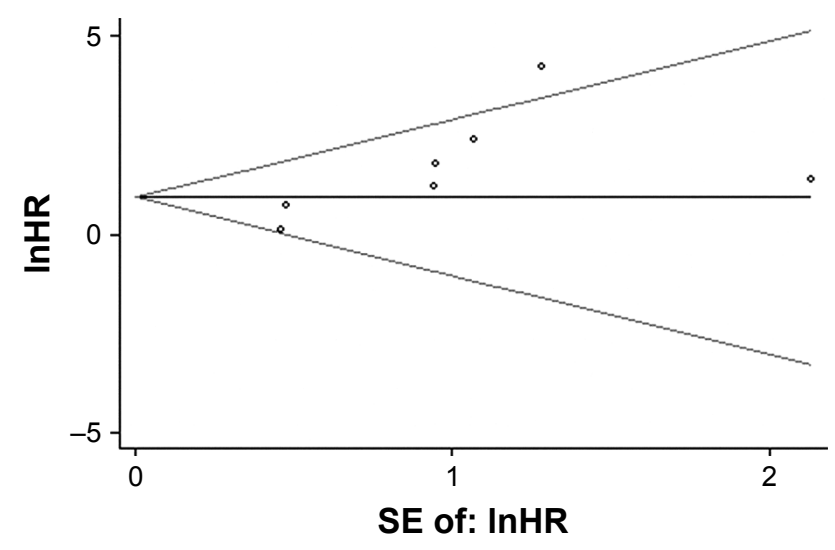

Figure 3 Funnel plots of publication bias on the association between pretreatment EBV-DNA and OS.

Abbreviations: EBV, Epstein-Barr virus; OS, overall survival; SE, standard error of the mean; HR, hazard ratio.

outcomes brought by age, LDH level, Ann Arbor stage, etc. might be overcome by new treatment. ${ }^{14} \mathrm{EBV}$-DNA is derived from necrotic or apoptotic tumor cells, and thus it is strongly associated with prognosis of NKTCL patients. Previous studies found that pretreatment EBV-DNA levels can reflect the tumor burden. ${ }^{15-17}$ Therefore, using EBV-DNA as a prognostic biomarker for NKTCL is reasonable and significant.

In 2009, Kim et $\mathrm{al}^{16}$ reported the prognostic value of pretreatment EBV-DNA in the whole blood for patients with NKTCL. In Ito et al's ${ }^{11}$ study, besides finding the significant association between pretreatment EBV-DNA and OS in NKTCL, consistent with the abovementioned result, they reported that pretreatment EBV-DNA was frequently detected in whole blood samples. However, in a 2016 study, researchers found that comparing with peripheral blood mononuclear cells, plasma appears to be a better specimen source when evaluating for $\mathrm{EBV}+$ disease response to therapy. ${ }^{26}$ However, Kim et $\mathrm{al}^{27}$ found that the proportion of patients with positive pretreatment EBV-DNA was not related to the type of sample tested (ie, plasma vs whole blood; $p=0.51$ ). Moreover, EBV-DNA was initially regarded as an independent risk factor in a prognostic model, which was established for patients with NKTCL in Kim et $\mathrm{al}^{\prime} \mathrm{s}^{27}$ study. The results of our subgroup analysis stratified by sample type were consistent with these previous studies. However, Tse and Kwong ${ }^{28}$ thought that whole blood was not the suitable material: first, because the quantitative mean of EBV-DNA level varies according to the leukocyte count; and second, circulating long-lived memory B cells infected with EBV may interfere with the results of EBV-DNA testing. The study by Wang et a ${ }^{17}$ suggested that pretreatment EBV-DNA level independently had no impact on prognosis for the early stage NKTCL patients, but the posttreatment plasma EBVDNA level could predict early relapse and poor prognosis. ${ }^{17}$ Similarly, the multivariate analysis by Kwong et $\mathrm{al}^{29}$ showed

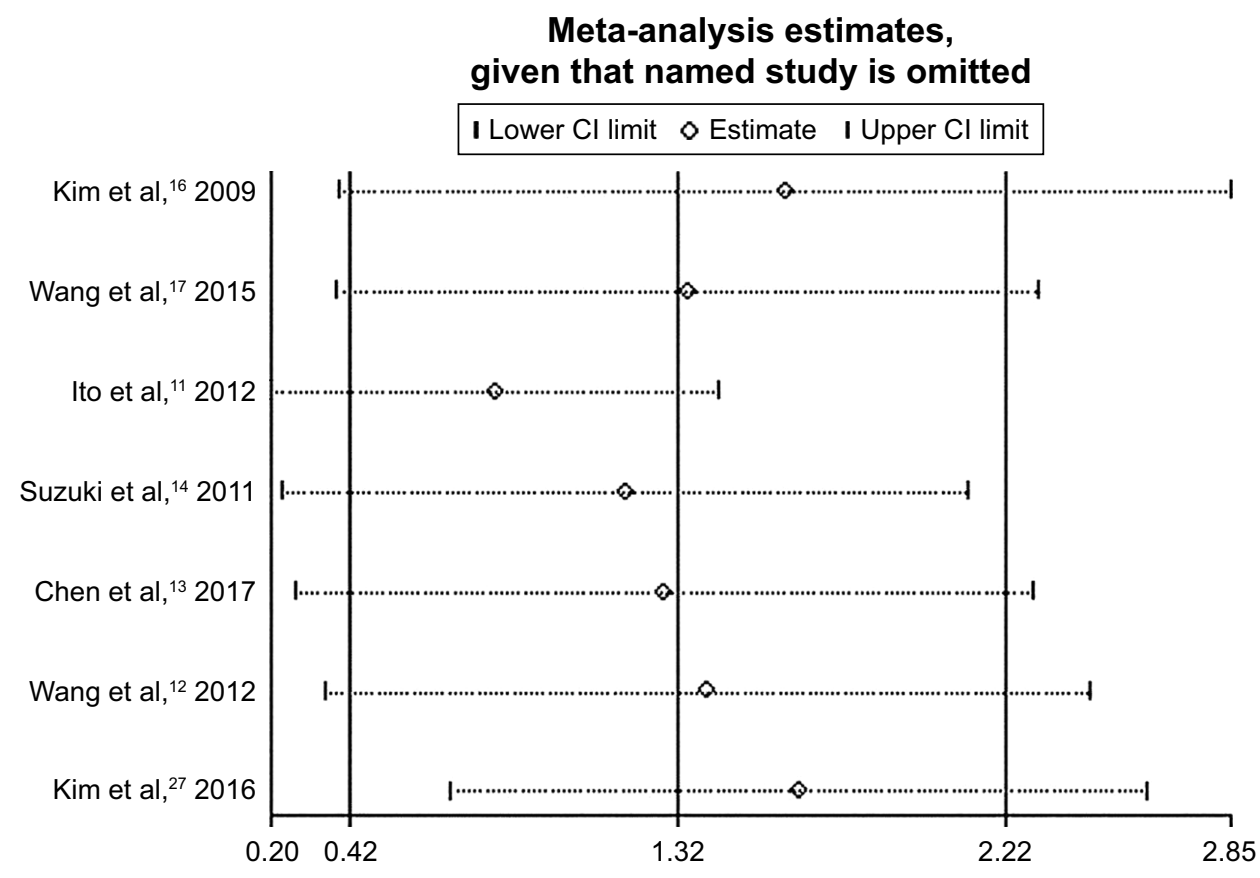

Figure 4 Sensitivity analysis on the association between pretreatment EBV-DNA and OS. Abbreviations: EBV, Epstein-Barr virus; OS, overall survival; $\mathrm{Cl}$, confidence interval. 
that pretreatment EBV-DNA levels, although accurate as a tumor load biomarker, did not independently affect survival; the changes in EBV-DNA after SMILE (dexamethasone, methotrexate, ifosfamide, petrylase, etoposide) treatment might be better to indicate tumor chemosensitivity and thus influence survival. The inconsistent prognostic value of pretreatment EBV-DNA might be related to the different chemotherapy regimens in different studies. In the studies by Wang et $\mathrm{al}^{17}$ and Kwong et al, ${ }^{29}$ all patients were treated with asparaginase-based therapy, whereas some patients in our meta-analysis were given conventional chemotherapy, such as CHOP (cyclophosphamide, doxorubicin, vincristine, prednisone) and CHOP-like regimens. Previous studies showed that patients with NKTCL have poor responses to anthracyclinecontaining chemotherapy because of the multidrug-resistant P-glycoprotein expressed by natural killer cells. ${ }^{30-32}$ Therefore, whether patients received anthracycline-containing chemotherapy was considered as a factor that could confound the assessment of prognostic value of pretreatment EBV-DNA.

Some limitations exist in our analysis. First, a significant heterogeneity was observed within the studies. Subgroup analyses were further performed. Results revealed that cutoff values might contribute to the heterogeneity. Second, all patients in this meta-analysis were Asian. However, ethnic bias is likely to be small because the universal association of NKTCL with EBV across all ethnic groups suggests a common pathogenesis. ${ }^{33}$ Third, some HRs and 95\% CIs were not directly extracted from the studies but were calculated according to Kaplan-Meier survival curves. Hence, the errors were inevitable. However, we strictly followed the methods provided by Tierney et al to extract survival rates from OS curves. Extraction for each OS curve was repeated three times to minimize errors to a great extent. Fourth, not all of the patients in our analysis received asparaginase-based chemotherapy. Different types of chemotherapy regimens lead to different survival outcomes, which may influence our final results. Fifth, at present, no consensus exists for the most appropriate cut-off value to be used for the measurement of EBV-DNA. The subgroup analysis results of this study indicated that the cut-off value may affect the final results of analysis. Therefore, the results of the analysis should be re-evaluated if the issues related to the cut-off value, to be used for the EBV-DNA testing, are resolved in future studies. Moreover, considering the rarity of this disease, the sample size of eligible studies was not large enough, which might be one of the reasons for the $p$-value $=0.05$ in the results of the $\mathrm{Q}$ test and $I^{2}$ text. Therefore, more large-scale studies should be performed to further validate the results.

\section{Conclusion}

The results of this meta-analysis suggested that pretreatment EBV-DNA positivity had an adverse effect on the survival outcome of NKTCL, despite these limitations mentioned herein before. Clinicians can formulate specific treatment regimens for NKTCL patients based on pretreatment EBVDNA levels. In addition, further large-scale studies regarding prognostic value of pretreatment EBV-DNA in NKTCL should be conducted to validate our findings in the era of asparaginase-based chemotherapy.

\section{Acknowledgment}

This research was supported by grants from the Six Major Talent Peak Project of Jiangsu Province (class B, no WS-047).

\section{Author contribution}

QF, XKT, and LY designed the systematic review; WJZ, YW, FYP, HMZ, and JW were involved in the literature search and study selection; WJZ, YW, FYP, HMZ, and JW extracted the data from eligible studies; QF and XKT conducted the analysis; QF, XKT, LY, and XH were involved in the interpretation of the results. QF, XKT, LY, and XH were responsible for the writing and critical revisions of the manuscript. All authors read and approved the final manuscript. All authors contributed toward data analysis, drafting and revising the paper and agree to be accountable for all aspects of the work.

\section{Disclosure}

The authors report no conflicts of interest in this work.

\section{References}

1. Kwong YL. Natural killer-cell malignancies: diagnosis and treatment. Leukemia. 2005;19(12):2186-2194.

2. Sun J, Yang Q, Lu Z, et al. Distribution of lymphoid neoplasms in China: analysis of 4,638 cases according to the World Health Organization classification. Am J Clin Pathol. 2012;138(3):429-434.

3. Yang QP, Zhang WY, Yu JB, et al. Subtype distribution of lymphomas in Southwest China: analysis of 6,382 cases using WHO classification in a single institution. Diagn Pathol. 2011;6:77.

4. Liu J, Song B, Fan T, et al. Pathological and clinical characteristics of 1,248 non-Hodgkin's lymphomas from a regional cancer hospital in Shandong, China. Asian Pac J Cancer Prev. 2011;12(11):3055-3061.

5. Kim SJ, Kim K, Kim BS, et al. Phase II trial of concurrent radiation and weekly cisplatin followed by VIPD chemotherapy in newly diagnosed, stage IE to IIE, nasal, extranodal NK/T-Cell Lymphoma: Consortium for Improving Survival of Lymphoma study. J Clin Oncol. 2009;27(35):6027-6032.

6. Yamaguchi M, Tobinai K, Oguchi M, et al. Phase I/II study of concurrent chemoradiotherapy for localized nasal natural killer/T-cell lymphoma: Japan Clinical Oncology Group Study JCOG0211. J Clin Oncol. 2009;27(33):5594-5600.

7. Kim SJ, Yang DH, Kim JS, et al. Concurrent chemoradiotherapy followed by L-asparaginase-containing chemotherapy, VIDL, for localized nasal extranodal NK/T cell lymphoma: CISL08-01 phase II study. Ann Hematol. 2014;93(11):1895-1901. 
8. Lei KI, Chan LY, Chan WY, Johnson PJ, Lo YM. Quantitative analysis of circulating cell-free Epstein-Barr virus (EBV) DNA levels in patients with EBV-associated lymphoid malignancies. Br J Haematol. 2000; 111(1):239-246.

9. Chan KC, Zhang J, Chan AT, et al. Molecular characterization of circulating EBV DNA in the plasma of nasopharyngeal carcinoma and lymphoma patients. Cancer Res. 2003;63(9):2028-2032.

10. Kimura H, Ito Y, Suzuki R, Nishiyama Y. Measuring Epstein-Barr virus (EBV) load: the significance and application for each EBV-associated disease. Rev Med Virol. 2008;18(5):305-319.

11. Ito Y, Kimura H, Maeda Y, et al. Pretreatment EBV-DNA copy number is predictive of response and toxicities to SMILE chemotherapy for extranodal NK/T-cell lymphoma, nasal type. Clin Cancer Res. 2012; 18(15):4183-4190.

12. Wang ZY, Liu QF, Wang $\mathrm{H}$, et al. Clinical implications of plasma Epstein-Barr virus DNA in early-stage extranodal nasal-type NK/T-cell lymphoma patients receiving primary radiotherapy. Blood. 2012; 120(10):2003-2010.

13. Chen Y, Zheng X, Chen B, et al. The clinical significance of EpsteinBarr virus DNA in peripheral blood mononuclear cells in patients with non-Hodgkin lymphoma. Leuk Lymphoma. 2017;58(10):2349-2355.

14. Suzuki R, Yamaguchi M, Izutsu K, et al. Prospective measurement of Epstein-Barr virus-DNA in plasma and peripheral blood mononuclear cells of extranodal NK/T-cell lymphoma, nasal type. Blood. 2011;118(23) 6018-6022.

15. Kim HS, Ryu KJ, Ko YH, et al. Macrophage inflammatory protein 1 alpha (MIP-1 $\alpha$ ) may be associated with poor outcome in patients with extranodal NK/T-cell lymphoma. Hematol Oncol. 2017;35(3):310-316.

16. Kim HS, Kim KH, Kim KH, et al. Whole blood Epstein-Barr virus DNA load as a diagnostic and prognostic surrogate: extranodal natural killer/T-cell lymphoma. Leuk Lymphoma. 2009;50(5):757-763.

17. Wang L, Wang H, Wang JH, et al. Post-treatment plasma EBV-DNA positivity predicts early relapse and poor prognosis for patients with extranodal NK/T cell lymphoma in the era of asparaginase. Oncotarget. 2015;6(30):30317-30326.

18. Moher D, Liberati A, Tetzlaff J, Altman DG. Reprint - preferred reporting items for systematic reviews and meta-analyses: the PRISMA statement. Phys Ther. 2009;89(9):873-880.

19. Moher D, Liberati A, Tetzlaff J, Altman DG. Preferred reporting items for systematic reviews and meta-analyses: the PRISMA statement Int J Surg. 2010;8(5):336-341.
20. Royston P, Parmar MK. Restricted mean survival time: an alternative to the hazard ratio for the design and analysis of randomized trials with a time-to-event outcome. BMC Med Res Methodol. 2013;13:152.

21. Parmar MK, Torri V, Stewart L. Extracting summary statistics to perform meta-analyses of the published literature for survival endpoints. Stat Med. 1998;17(24):2815-2834.

22. Stang A. Critical evaluation of the Newcastle-Ottawa scale for the assessment of the quality of nonrandomized studies in meta-analyses. Eur J Epidemiol. 2010;25(9):603-605.

23. Tierney JF, Stewart LA, Ghersi D, Burdett S, Sydes MR. Practical methods for incorporating summary time-to-event data into meta-analysis. Trials. 2007;8:16

24. Higgins JP, Thompson SG. Quantifying heterogeneity in a metaanalysis. Stat Med. 2002;21(11):1539.

25. Higgins JP, Thompson SG, Deeks JJ, Altman DG. Measuring inconsistency in meta-analyses. BMJ. 2003;327(7414):557-560.

26. Kanakry JA, Hegde AM, Durand CM, et al. The clinical significance of EBV DNA in the plasma and peripheral blood mononuclear cells of patients with or without EBV diseases. Blood. 2016;127(16):2007.

27. Kim SJ, Yoon DH, Jaccard A, et al. A prognostic index for natural killer cell lymphoma after non-anthracycline-based treatment: a multicentre, retrospective analysis. Lancet Oncol. 2016;17(3):389.

28. Tse E, Kwong YL. The diagnosis and management of NK/T-cell lymphomas. J Hematol Oncol. 2017;10(1):85.

29. Kwong YL, Pang AW, Leung AY, Chim CS, Tse E. Quantification of circulating Epstein-Barr virus DNA in NK/T-cell lymphoma treated with the SMILE protocol: diagnostic and prognostic significance. Leukemia. 2014;28(4):865-870.

30. Yamaguchi M, Kita K, Miwa H, et al. Frequent expression of P-glycoprotein/MDR1 by nasal T-cell lymphoma cells. Cancer. 1995;76(11): 2351.

31. Kim WS, Song SY, Ahn YC, et al. CHOP followed by involved field radiation: is it optimal for localized nasal natural killer/T-cell lymphoma? Ann Oncol. 2001;12(3):349-352.

32. Lee SH, Ahn YC, Kim WS, Ko YH, Kim K, Park K. The effect of preirradiation dose intense CHOP on anthracyline resistance in localized nasal NK/T-cell lymphoma. Haematologica. 2006;91(3):427-428.

33. Haverkos BM, Pan Z, Gru AA, et al. Extranodal NK/T Cell Lymphoma, Nasal Type (ENKTL-NT): an update on epidemiology, clinical presentation, and natural history in North American and European cases. Curr Hematol Malig Rep. 2016;11(6):514. 


\section{Supplementary materials}

Table SI Search strategies for three electronic databases

\begin{tabular}{ll}
\hline Electronic database & Search strategy \\
\hline PubMed & (Extranodal NK-T-Cell Lymphoma[Title/ \\
& Abstract]) AND (prognosis[Title/Abstract] \\
& OR (EBV DNA[Title/Abstract])) \\
& 'nk t cell lymphoma':ab,ti OR \\
& 'extranodal nk/t cell lymphoma':ab,ti \\
EMBASE & AND 'prognosis':ab,ti AND ('epstein \\
& barr virus':ab,ti OR 'ebv':ab,ti) AND \\
& [embase]/lim \\
& (extranodal nk/t cell lymphoma) AND \\
& (epstein barr virus) AND prognosis \\
\hline
\end{tabular}

Table S2 Newcastle-Ottawa quality assessment scale

Assessment of quality of a cohort study - NewcastleOttawa Scale

Selection (tick one box in each section)

I. Representativeness of the intervention cohort

a) Truly representative of the average, elderly, communitydwelling resident

b) Somewhat representative of the average, elderly, community-dwelling resident

c) Selected group of patients, eg, only certain socioeconomic groups/areas

d) No description of the derivation of the cohort

2. Selection of the nonintervention cohort

a) Drawn from the same community as the intervention cohort

b) Drawn from a different source

c) No description of the derivation of the nonintervention cohort

3. Ascertainment of intervention

a) Secure record (eg, health care record)

b) Structured interview

c) Written self-report

d) Other/no description

4. Demonstration that outcome of interest was not present at start of study

a) Yes

b) No
Table S2 (Continued)

Assessment of quality of a cohort study - NewcastleOttawa Scale

Comparability (tick one or both boxes, as appropriate)

I. Comparability of cohorts on the basis of the design or analysis

a) Study controls for age, sex, marital status

b) Study controls for any additional factors (eg, socioeconomic status, education)

Outcome (tick one box in each section)

I. Assessment of outcome
a) Independent blind assessment
b) Record linkage
c) Self-report
d) Other/no description

2. Was follow-up long enough for outcomes to occur

a) Yes, if median duration of follow-up $\geq 6$ months

b) No, if median duration of follow-up $<6$ months

3. Adequacy of follow-up of cohorts

a) Complete follow-up: all subjects accounted for

b) Subjects lost to follow-up unlikely to introduce bias: number lost $\leq 20 \%$, or description of those lost suggesting no different from those followed

c) Follow-up rate $<80 \%$ (select an adequate $\%$ ) and no description of those lost

d) No statement
OncoTargets and Therapy

\section{Publish your work in this journal}

OncoTargets and Therapy is an international, peer-reviewed, open access journal focusing on the pathological basis of all cancers, potential targets for therapy and treatment protocols employed to improve the management of cancer patients. The journal also focuses on the impact of management programs and new therapeutic agents and protocols on

\section{Dovepress}

patient perspectives such as quality of life, adherence and satisfaction. The manuscript management system is completely online and includes a very quick and fair peer-review system, which is all easy to use. Visit http://www.dovepress.com/testimonials.php to read real quotes from published authors. 